\title{
Gender Effects in Physics Assessments of Kinematic Graphs
}

\section{Dr. Robert A Ross, University of Detroit Mercy}

Robert A. Ross is a Professor of Physics in the Department of Chemistry \& Biochemistry at the University of Detroit Mercy. His research interests include semiconductor devices, photovoltaics, and physics pedagogy. Ross received his B.S. and Ph.D. degrees in Physics from Wayne State University in Detroit.

Dr. Katherine Snyder, University of Detroit Mercy 


\section{Gender Effects in Physics Assessments of Kinematic Graphs}

\section{Introduction}

In a previous publication we presented an argument that by organizing student learning outcomes and course objectives around the principle of the operational definition of technical terms that the assessment of student learning can in some ways be simplified. By operational definition we mean that students are describing a process, or operation, to give meaning to technical terms. For example we can give an operational meaning to "derivative", by describing the following operation: drawing tangent lines on a position versus time graph at regular intervals of time, examine the slope of each tangent line segment and ask if it is positive or negative, and large or small, plot the value of the slopes of the tangent lines and generate a velocity versus time graph. We also believe that by evaluating how students perform these operations we can determine a particular student's strengths and weaknesses, and as a consequence develop strategies to improve instruction.

There are numerous learning outcomes in our introductory physics courses. In this paper we will discuss two. One stated outcome is to "compare written, algebraic, and graphical descriptions of motion." This outcome is meant to have students use various representations to describe motion. Students could observe a ball rolling on a track and state, "The ball started out rolling fast in the positive direction. It slowed down, stopped, and reversed direction and subsequently sped up while travelling in the negative direction.” The description should be complete enough to allow someone reading it to understand what was observed. Students could draw pictures that represent stroboscopic photographs; sketches of the location of the object at equal intervals of time. In addition, they could generate graphs of position (or velocity or acceleration) versus time. Students should be able to observe the motion of an object and determine what variables to use to describe the motion, e.g. whether it has a constant velocity or a constant acceleration and to use an equation to relate the relevant variables. The goal is to give students' the ability to transition between the three modes of representation. An additional goal is to have students recognize the relationships between graphical representations of position, velocity, and acceleration functions in terms of derivatives and integrals.

For 19 years students in the introductory calculus-based physics course at the University of Detroit Mercy were assessed using a variation of a problem introduced by Arons ${ }^{1}$ in Part II of his text. In a previous work on using operational definitions, we examined the results of this assessment for 8 sections of the introductory course. In this paper we extend the work and disaggregate the student population by gender. We provide students a chance to cooperatively correct examinations. We believe this practice improves student learning and has implications for the administration of fair and equitable assessments of that learning. 


\section{Student population}

The University of Detroit Mercy is a comprehensive Catholic university founded upon the Jesuit and Mercy educational traditions. The engineering program began in 1911 and the University currently offers programs in Architectural, Civil, Electrical \& Computer, Environmental, Mechanical, Software, and Robotics and Mechatronics Systems Engineering. The university operates on a semester basis and incoming engineering students take calculus in the fall and general physics I in the winter of their freshman year. They subsequently take general physics II in the fall semester of their sophomore year, depending on mathematics placement and successful completion of prerequisites.

\section{Graphical Representations: The Problem}

The problem involves student responses to a set of questions regarding 6 different velocity versus time graphs. Student performance on these questions indicates they are quite challenging for students. So as not to use the same question term after term; the questions and graphs have been modified each semester, with pertinent features maintained. For instance, the graphs remain straight line segments so interpreting the value or the slope should be of consistent difficulty. Straight line segments also allow for the area under each segment of a graph, the displacement, to be a combination of the area of a triangle and a rectangle. Secondly, the questions are varied but in such a way as to keep the level of difficulty consistent. For example one term we may ask "Which object (or objects) are always speeding up?" and the next term something like "Which object (or objects) never speed up?”

We believe that the time and effort spent on analyzing these graphs has significant value because as students' progress though the curriculum, the graphical representations become more complex. For example the spectral radiance involves a variable like wavelength on the horizontal axis but the vertical axis is a derivative with unfamiliar units and interpretation. In an upper level modern physics course we introduce the idea of a cumulative distribution functions in the following manner. Suppose we have a large population of people and need to determine some statistics of a particular physical feature such as height in $\mathrm{cm}, h$. We imagine setting up a pole with a bar at height $h$ and counting the number of people that fit underneath. We subsequently raise the bar and again count the number of people that fit underneath. Everyone recognizes that nobody is shorter than $10 \mathrm{~cm}$ and everyone is shorter than $300 \mathrm{~cm}$. As a result, a graph of the number of people, $N$, shorter than the height $h$ will look something like the plot on the left in Fig. 1 . The derivative of this function with respect to $h$, gives us another distribution function that can be used to answer a variety of useful questions about the average height, etc. We realize that there are also meaningless questions such as “How many people are $150 \mathrm{~cm}$ tall?” We can only ask how many people are there within some height interval centered on a value. This is apparent when we look at how we count using the distribution function $N=\int_{h_{1}}^{h_{2}} d N=\int_{h_{1}}^{h_{2}} \frac{d N}{d h} d h$, if the limits are the same the integral vanishes. 

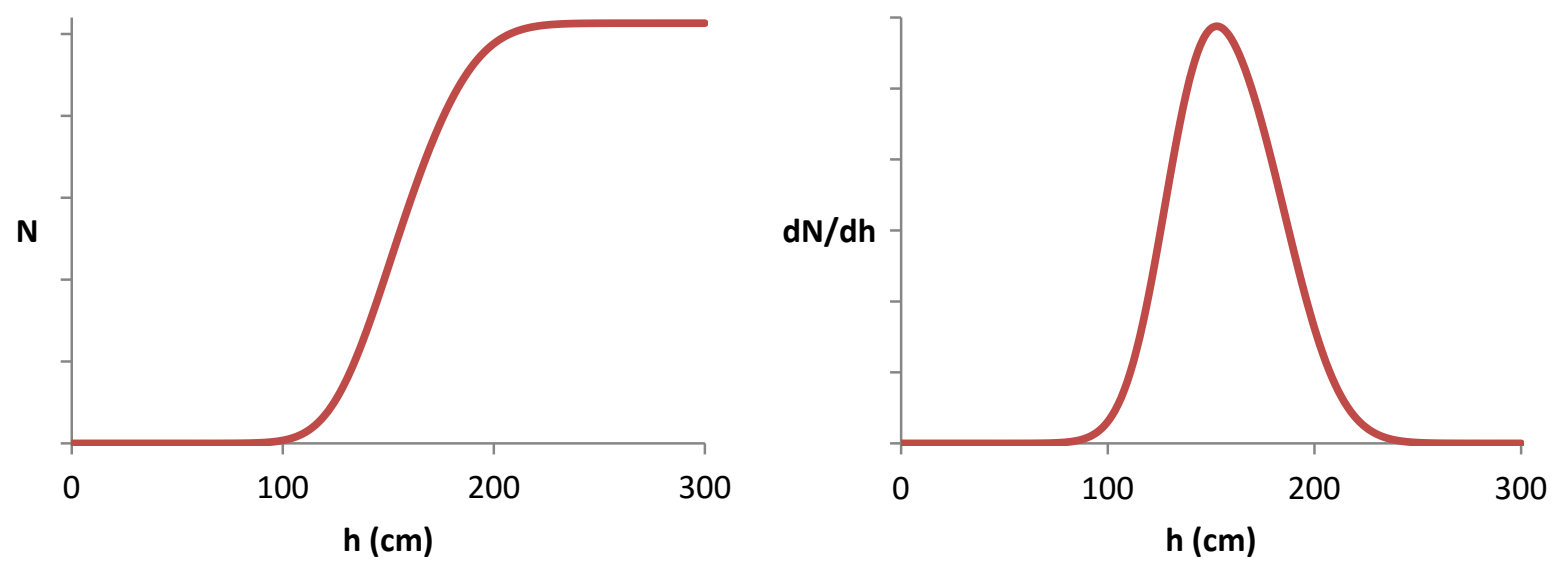

Figure 1

The graph on the left shows the number of people that are shorter than $h$. The graph on the right is the derivative of $N$ with respect to $h$.

This type of graph is important, because it allows us to build on this prior knowledge when approaching a less familiar context. For instance, if we replace the "number of people shorter than height $h$ " with the "total intensity of light with wavelength less than $\lambda$ " in nm; which could be measured on a pattern of light that is transmitted through a diffraction grating or prism. We would end up with a graph that looks qualitatively like the left one in Fig. 1 except the vertical axis would be $\mathrm{W} / \mathrm{m}^{2}$ and the horizontal axis would be wavelength in nm. If we differentiate that graph with respect to wavelength we end up with a spectral radiance function with units on the vertical axis of $W / \mathrm{m}^{2} / \mathrm{nm}$. Can we ask, “What is the intensity at 550nm?" If students' cannot recognize the relationships between these graphs, then interpreting spectra becomes an almost intractable problem. Over the past few years students in a modern physics course were asked to explain the units of the $d N / d h$ graph. Only about $5 \%$ were successful.

Researchers in physics education have recognized for a long time that students have difficulties interpreting the values, slopes and areas of graphs in the context of kinematics. ${ }^{2}$ One of the observations mentioned in Ref. 2 is that, "The errors we identified did not seem to be idiosyncratic to any particular group but were evident among different populations and across different levels of sophistication." Evidence of a marked disconnect between elementary calculus concepts and physics students' abilities to interpret graphical representations have been noted with a wide variety of student populations including honors courses. ${ }^{3,4}$ Multiple choice tests, e.g. Test of Understanding Graphs in Kinematics (TUG-K) have been developed and rigorously analyzed to test large numbers of students using objective assessments. ${ }^{5}$ An analysis of the TUGK results shown in Ref. 5 indicate that the mean score for males is 9.5 (out of 21 or $45 \%$ ) while that for females is 7.2 (34\%), a difference deemed significant. We will see that our results are remarkably similar. In that paper the author concludes that, "Students need to understand graphs before they can be used as a language for instruction. Teachers may want to utilize Arons' idea of operationally defining kinematic concepts." ${ }^{\prime 6}$ In our instructional practice we believe strongly in the idea of operational definition and use it as a foundational concept. ${ }^{7}$

For the past 19 years we have posed a set of similar problems to all incoming engineering and science students taking introductory physics. The problems involve the analysis of velocity as a 
function of time graphs, $v(t)$, for the one dimensional motion of six identical objects. These problems are based upon the work of Arons found in Ref.1. Using graphs of velocity as a function of time allows us to ask questions regarding information that can be directly obtained from the graph, as well as what can be gleaned from the derivative and integral of the graph. As an example we show below in Fig. 2 the graphs given to students during the winter term of 2015.

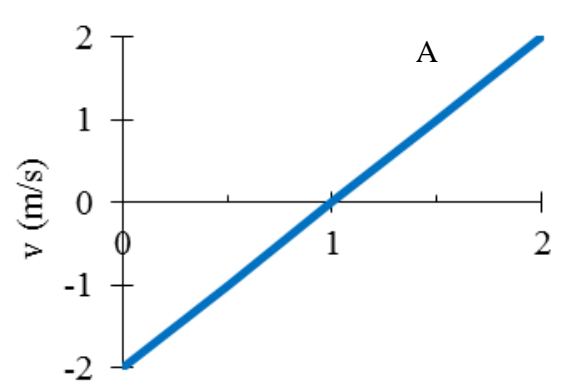

$\mathrm{t}(\mathrm{s})$

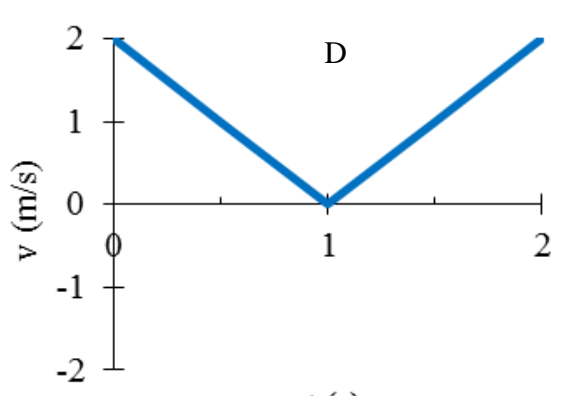

$\mathrm{t}(\mathrm{s})$

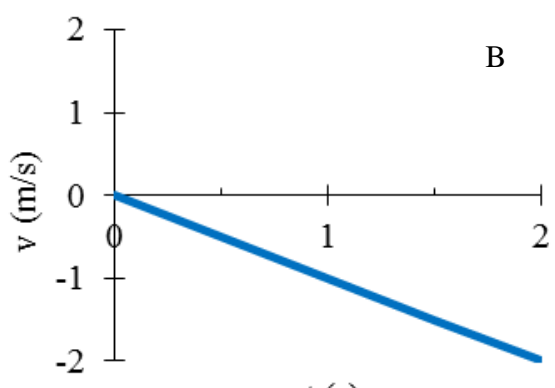

$t(s)$

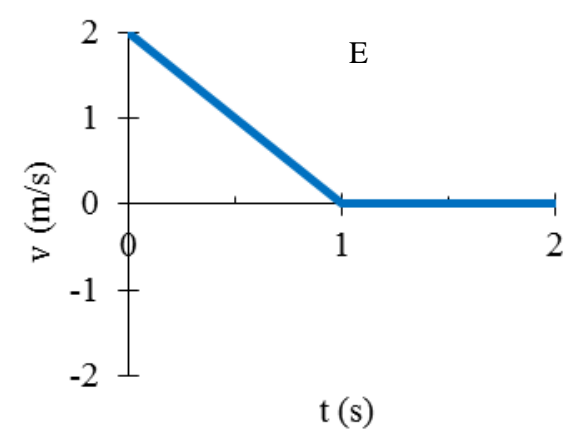

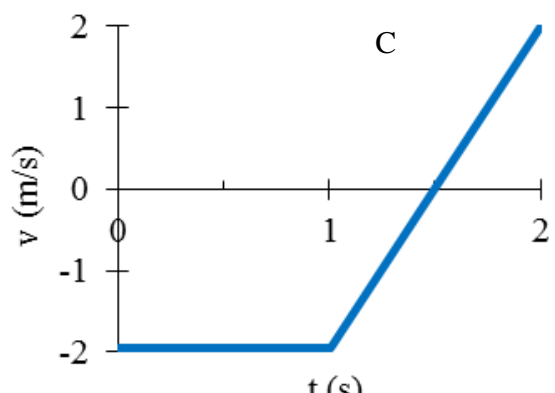

$\mathrm{t}(\mathrm{s})$

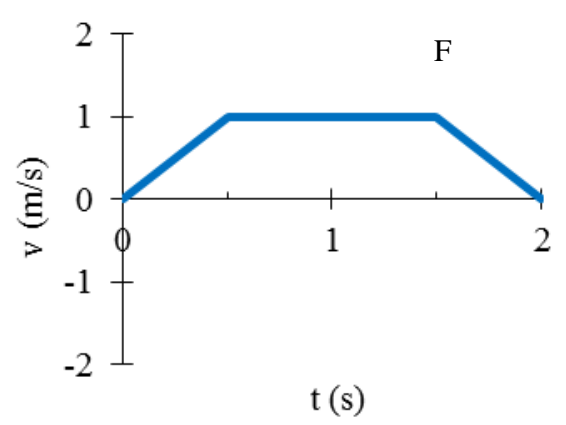

Figure 2

Sample velocity versus time graphs used in the first exam and in the final exam.

We asked a set of questions to all of the students on the first exam in the class. In addition, on the final examination students were shown the same graphs and asked to answer additional questions. Some of the questions on the final exam were the same as the first exam and other questions were included that involved concepts more indirectly related to the graphs such as momentum, changes in momentum, work, kinetic energy, changes is kinetic energy, and force. The questions asked last year are reproduced below for clarity. Students are prompted to circle the correct responses on a line shown here:
A $\quad$ B $\quad$ C
D E F
NONE.

In order to correctly answer the majority of the questions, students would need to circle more than one letter. All correct responses need to be marked to receive a point, there is no partial credit.

On the last page are six velocity versus clock reading histories which describe the one dimensional motion of six objects that started out from the origin $x=0 \mathrm{~m}$ at time $t=0 \mathrm{~s}$. Circle the correct answer(s) for each of the following questions.

(a) Which object (or objects) are located at the origin, $x=0$, at the clock reading $t=2 \mathrm{~s}$ ?

(b) Which object (or objects) spends at least some time moving in the negative direction? 
(c) Which object (or objects) moves with a constant, nonzero acceleration during the entire time interval?

(d) Which object (or objects) exhibits an acceleration that decreases?

(e) Which object (or objects) is closest to, but not at the origin, after 2s?

(f) Which object (or objects) was at rest for some period of time?

(g) Which object (or objects) changed direction?

(h) Which object (or objects) exhibits the smallest average velocity over the entire time period?

(i) Which object (or objects) started moving in the negative $x$ direction and then reversed the direction of motion, traveling back in the positive $x$ direction?

(j) Which object (or objects) never slowed down?

(k) Which object (or objects) slowed down and then sped up?

(l) Which object (or objects) are farthest away from the origin at $t=2 \mathrm{~s}$ ?

Questions on the final exam:

On the last page there are velocity versus clock reading histories which describe the one dimensional motion of six objects with the same mass that started out from position $x=0$ at time $t=0$ s. Circle the correct answer(s) for each of the following questions.

(a) Which object (or objects) moves under the influence of a constant (non-zero) net force?

(b) Which object (or objects) experiences a net force, at least some time, in the negative direction?

(c) Which object (or objects) have less kinetic energy at $t=2 \mathrm{~s}$ than at $t=0 \mathrm{~s}$ ?

(d) Which object (or objects) have net positive work done on them from $t=0 \mathrm{~s}$ to $t=2 \mathrm{~s}$ ?

(e) Which object (or objects) experience a force that changes in either magnitude or direction?

(f) Which object (or objects) is closest to, but not at, $x=0$ at the clock reading $t=2 \mathrm{~s}$ ?

(g) Which object (or objects) exhibits the smallest average velocity? (Be careful with the signs).

(h) Which two or more objects experience identical forces as a function of time? Identical $F(t)$ graphs.

(i) Which object (or objects) have $\Delta \mathrm{p}<0$ from $t=0$ s to $t=2 \mathrm{~s}$ ?

(j) Which object (or objects) never slowed down?

(k) Which object (or objects) experiences the greatest magnitude of force?

(l) Which object (or objects) experiences the largest average force from $t=0 \mathrm{~s}$ to $t=2 \mathrm{~s}$ ?

While we have given variations of these questions for 19 years we have only kept track of individual student responses since 2009. We analyzed the results for 9 sections of the course (it was not done for 3 terms due to an illness of the instructor.) The first exam was administered to a total of 194 students (38 females and 156 males) the final exam was taken by 188 students (38 females and 150 males). We place the scores in $20 \%$ bins to graphically represent the results. For example, if a student scored 60\% they are represented in the 60-80 bin. In Fig. 3 we show the number of students versus the percentage of correct responses. In Fig. 4 we show the percentages of female and male students and the corresponding percentage of correct responses. 


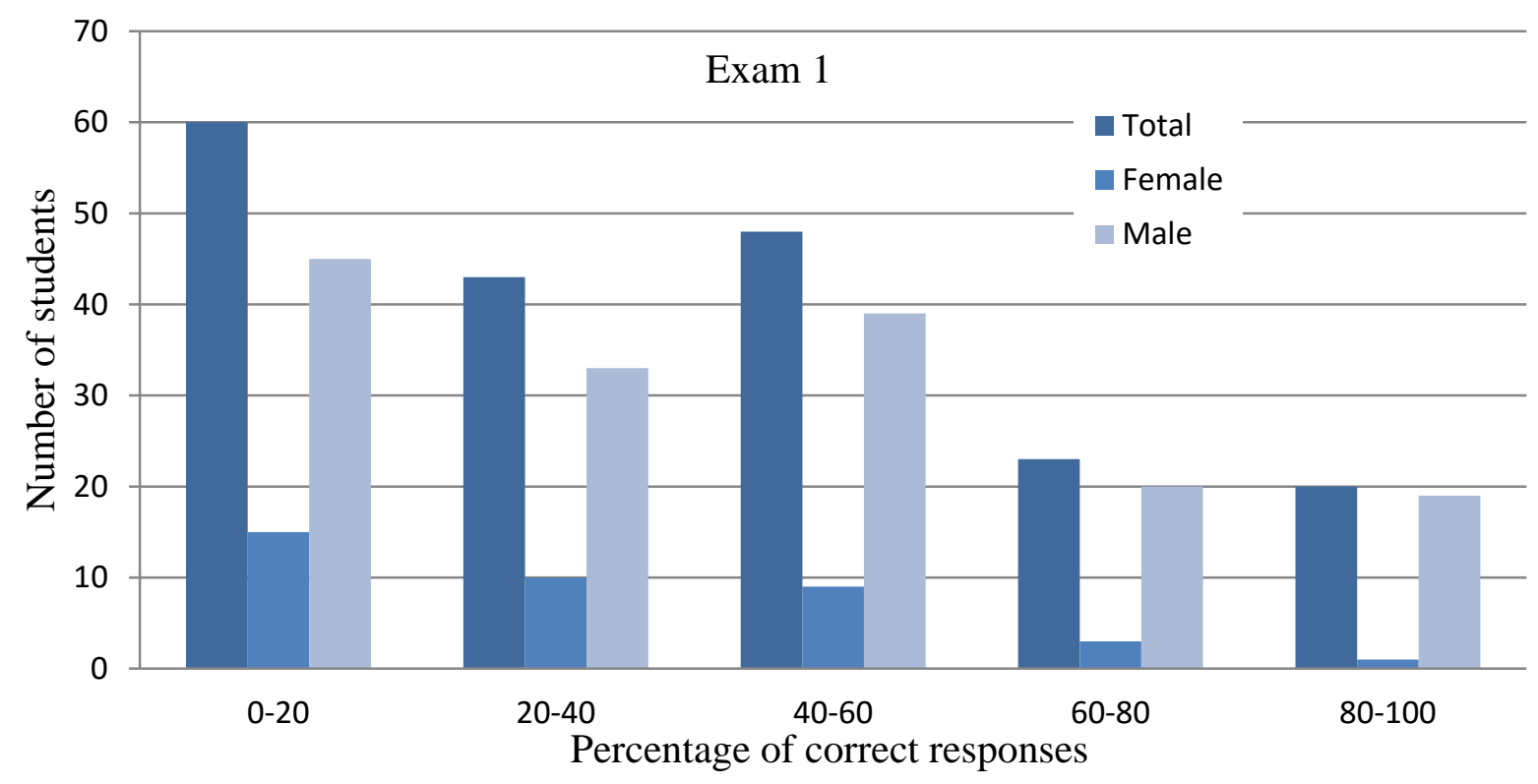

Figure 3

Number of students and percentage of correct responses.

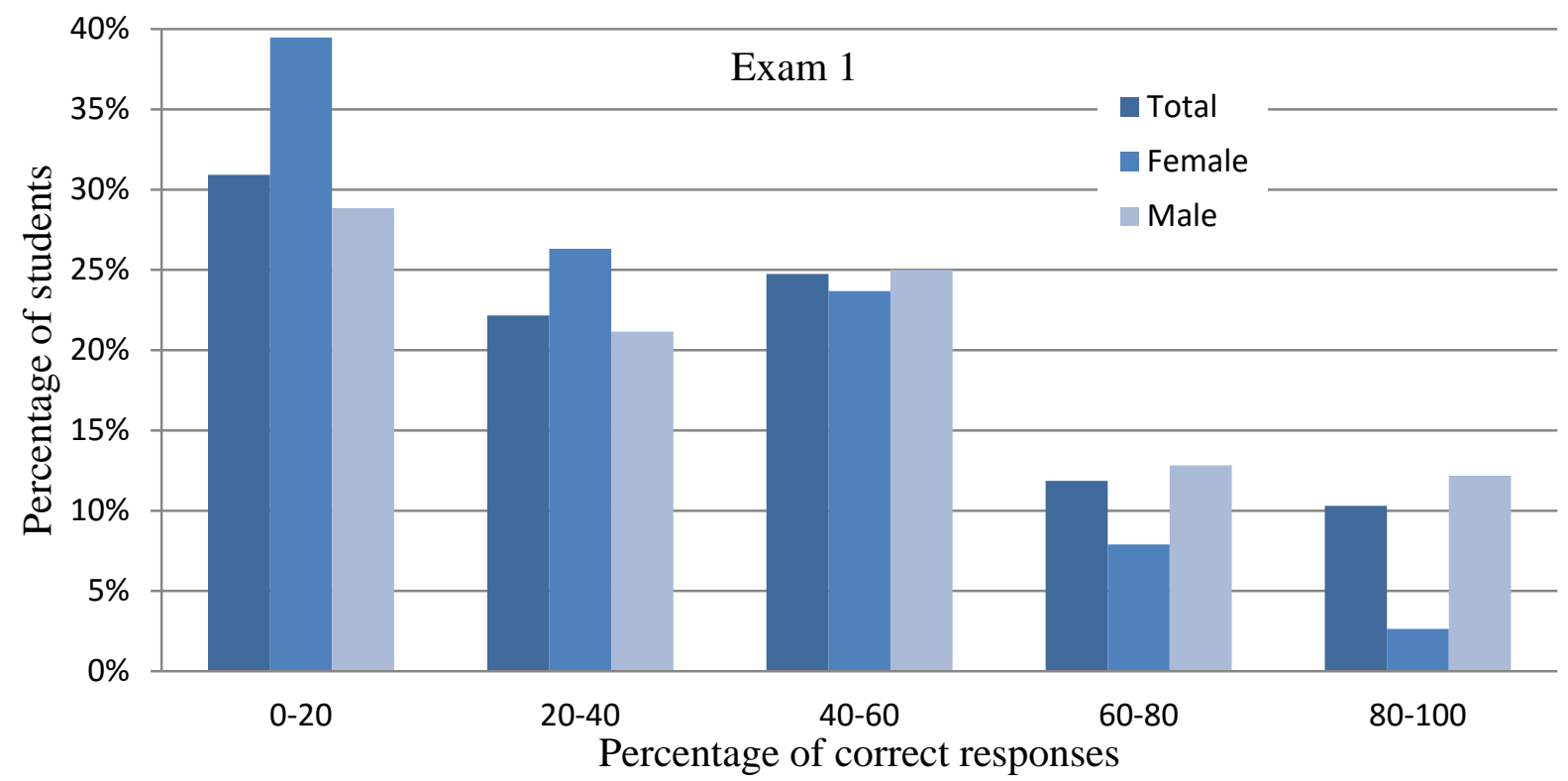

Figure 4

Percentage of female and male students and percentage of correct responses.

These data are somewhat more dramatic when we examine the numbers in each case as shown below in Table I. It is remarkable that only one female has scored in the top quintile since 2009. 
Test 1

\begin{tabular}{|c|c|c|c|}
\hline Bin & Total & Female & Male \\
\hline $0-20$ & 60 & 15 & 45 \\
\hline $20-40$ & 43 & 10 & 33 \\
\hline $40-60$ & 48 & 9 & 39 \\
\hline $60-80$ & 23 & 3 & 20 \\
\hline $80-100$ & 20 & 1 & 19 \\
\hline
\end{tabular}

Table I

Numbers of female and male students and percentage of correct responses from Test 1.

The overall percentage of correct scores given by females is $32 \%$ and the corresponding number for males is $40 \%$. These are remarkably consistent with the TUG-K results shown in Ref. 5 where the corresponding numbers are $34 \%$ and $45 \%$ respectively.

During the past 15 years we have engaged the students in a collaborative process of correcting the exams during the following meeting of the class. A typical examination consists of 3 pages of problems and a fourth sheet containing the relevant velocity graphs. Students are informed that the last page can be removed, but it is recommended that they keep it. During the exam students are allowed a 3x5 note card with any information they deem relevant. On the day of the corrections, clean copies of the first 3 pages are provided; they can correct all the problems on 2 of the pages. Students work in self-selected groups of 2 or 3 and are instructed that they are to discuss the solutions of the problems and not copy another students' work. When making corrections they are to explain the reasoning used to arrive at the solution. They are allowed to use their notes and the instructor is available to provide direction. A lively student discussion ensues and the instructor invariably hears comments like, "Now I understand! or "Of course, it makes sense!” These corrections have several advantages for the students. It allows students an opportunity to increases their course grade because the initial version and the corrected one are averaged together on a weighted basis. After the corrections are graded the instructor chooses a weighting factor to average the two grades. The first exam counts more than the corrected version because that was the original sample of the students' work. The corrected version is weighted so that the rank ordering of the student scores on the first attempt is not altered significantly after the weighted averaging. It does not seem fair for a student to end up with a higher ranking after making collaborative corrections. Using this process, all students that are present benefit. If students are absent on the day of the corrections their old exam score stands (except in instances where the student has been granted an exception by communicating an issue before the class meeting.) The students have an opportunity to learn from each other by discussing the answers and writing down their reasoning. As Redish points out, "explaining their reasoning" may not be sufficient and "think-aloud protocols" may be required. ${ }^{8}$ We believe, and we are not alone ${ }^{9}$, that allowing students to rework the exams and have meaningful discussions that effect their grades has an opportunity to improve learning. One additional consequence of this strategy is that student attendance improves significantly. Shown below in Figs. 5 and 6 are graphs of the results from the final exam. The student numbers are shown below in Table II. 


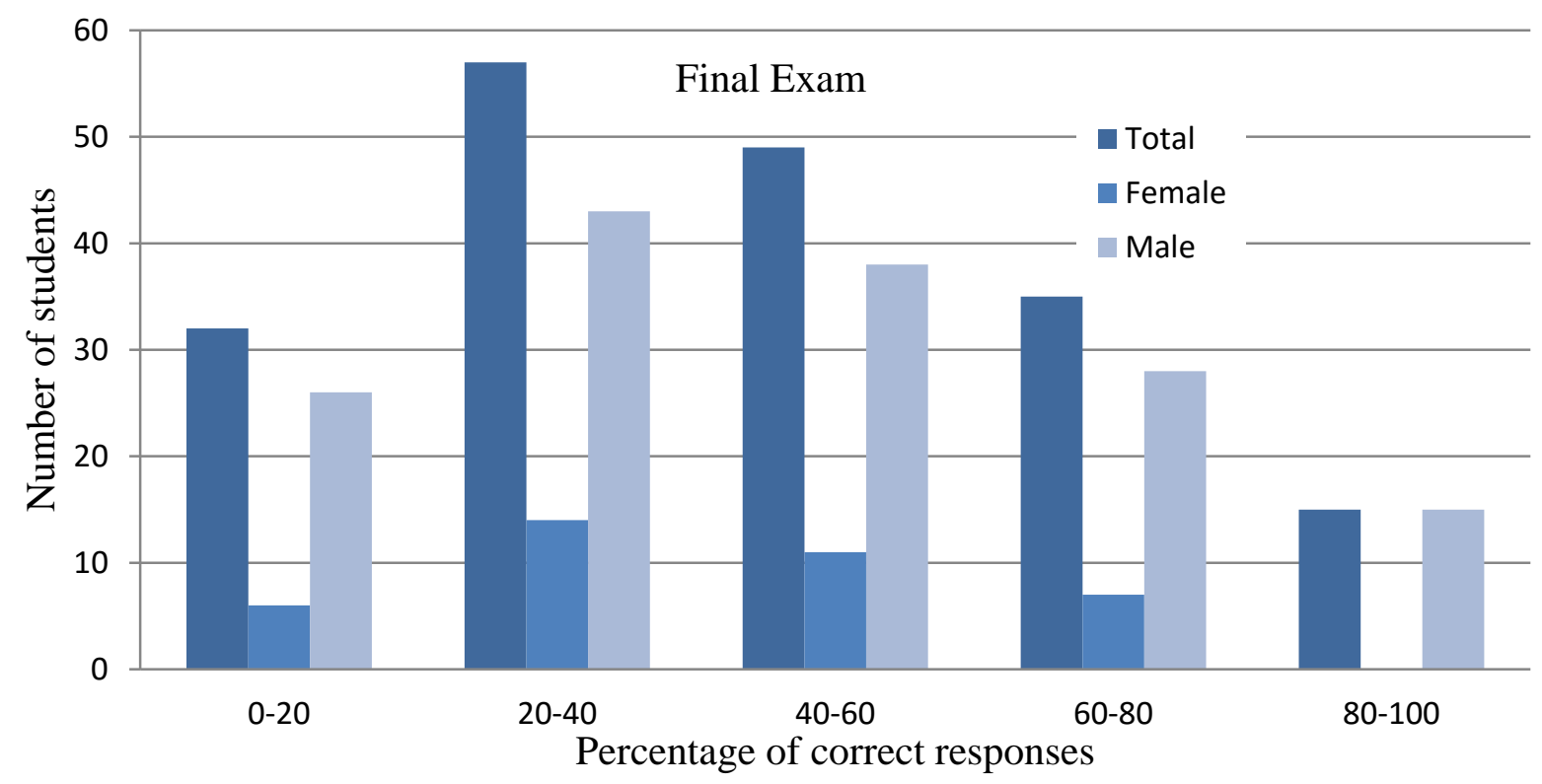

Figure 5

Number of students and percentage of correct responses.

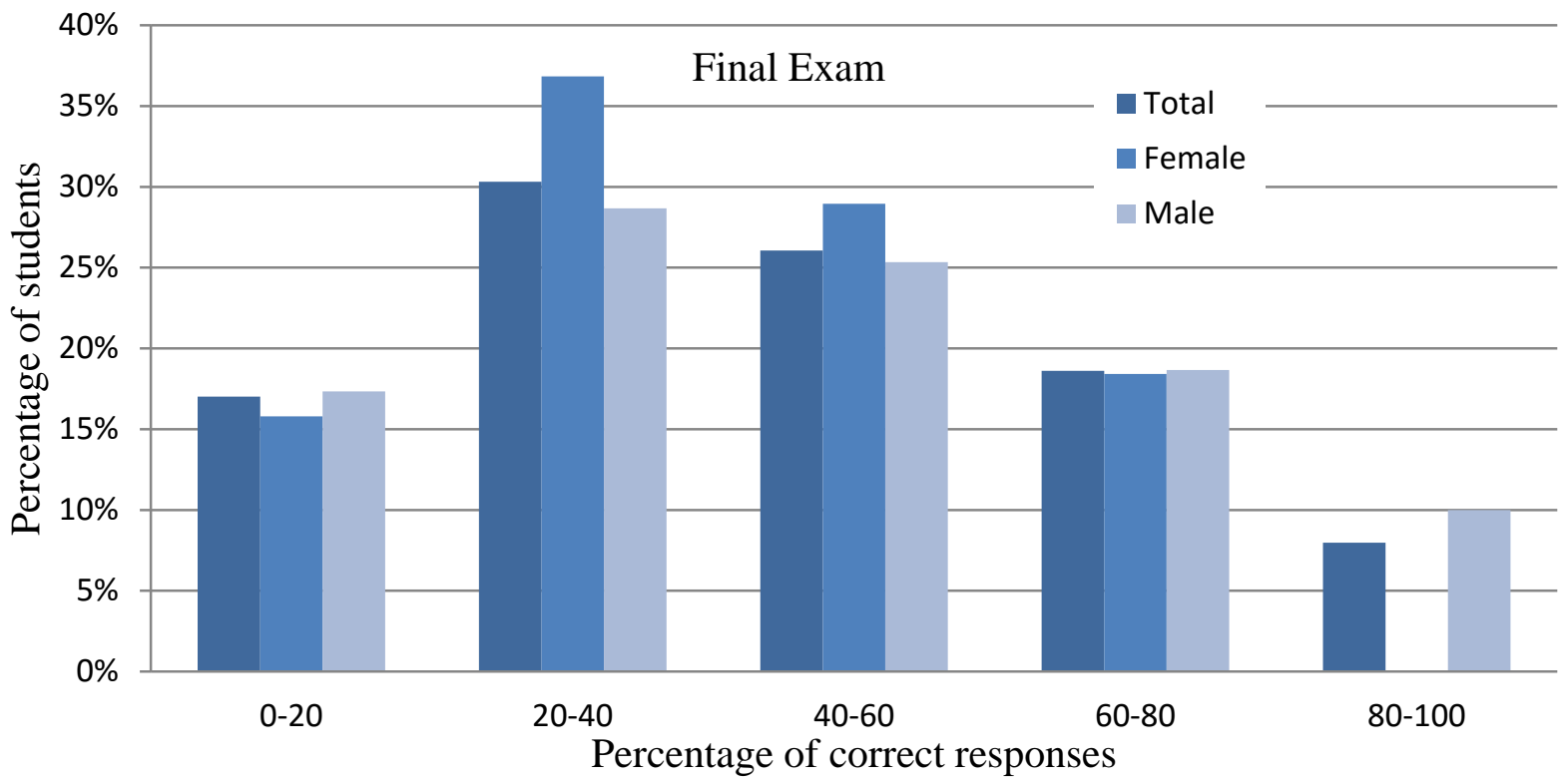

Figure 6

Percentage of female and male students and percentage of correct responses. 
Final Exam

\begin{tabular}{|c|c|c|c|}
\hline Bin & Total & Female & Male \\
\hline $0-20$ & 32 & 6 & 26 \\
\hline $20-40$ & 57 & 14 & 43 \\
\hline $40-60$ & 49 & 11 & 38 \\
\hline $60-80$ & 35 & 7 & 28 \\
\hline $80-100$ & 15 & 0 & 15 \\
\hline
\end{tabular}

Table II

Numbers of female and male students and percentage of correct responses from the final exam.

On the final exam the overall percentage of correct scores given by females is $38 \%$ and the corresponding number for males is $41 \%$. It is still the case the no females are in the upper quintile, however the distribution of scores has changed so that a significant number of females moved from the bottom quintile to the middle. We view this as an improvement in overall achievement by females on this particular assessment. The value of a paired, one-tailed Student's t-test is 0.045 . While the questions on the final exam are somewhat more challenging, there are gains made especially by female students. We believe that part of the gains for the female students is that they had an opportunity to reason and discuss, out loud, how they thought about a particular problem.

\begin{tabular}{|c|c|c|}
\cline { 2 - 3 } \multicolumn{1}{c|}{} & First Exam & Final Exam \\
\hline Females & $32 \%$ & $38 \%$ \\
\hline Males & $40 \%$ & $41 \%$ \\
\hline
\end{tabular}

Table III

Comparison of female and male students and percentages of correct responses from the first exam and from the final exam.

We plan to further investigate these interesting results. Over the next several weeks we intend to conduct student interviews to help determine how female and male students approach the problem of the graphical representation of kinematic data. We also would like to understand how student communication during the exam correction activities is viewed by different populations. We will present our results at a future meeting.

\section{Conclusion and Acknowledgement}

The achievement gap of female students from their male counterparts in physics is a vexing and serious problem. The future of our discipline depends on our ability to attract and retain female and underrepresented minority students. We believe that allowing students to work cooperatively to perform test corrections benefits all students but seems to increase the performance of females to a greater extent.

We are thankful for the thoughtful comments of the anonymous reviewers. Their comments resulted in an improved manuscript. 


\section{References}

1 A. Arons, Teaching Introductory Physics, John Wiley \& Sons, New York, 1997. (Part II Kinematics problem 2.11, pg. 15-16.)

2 L.C. McDermott, M.L. Rosenquist, and E.H. van Zee, "Student difficulties in connecting graphs and physics: Examples from kinematics," Am. J. Phys. 55, 503-513 (1987); doi: 10.1119/1.15104.

3 D.E. Trowbridge and L.C. McDermott, "Investigation of student understanding of the concept of velocity in one dimension," Am. J. Phys. 48, 1020-1028 (1980); http://dx.doi.org/10.1119/1.12298.

4 P. C. Peters, "Even honors students have conceptual difficulties with physics," Am. J. Phys. 50, 501-508, (1982); doi: 10.1119/1.12797.

5 R.J. Beichner, "Testing student interpretation of kinematics graphs," Am. J. Phys. 62, 750-762 (1994); doi: 10.1119/1.17449.

${ }^{6}$ Beichner refers to: A. Arons, A Guide to Introductory Physics Teaching, John Wiley \& Sons, New York, 2000, especially the first three chapters.

7 R. Ross, “Operational Definition and Assessment,” in Proceedings of the 2015 American Society for Engineering Education Annual Conference \& Exposition, Seattle, WA, (2015)

8 E.F. Redish, “Oersted Lecture 2013: How should we think about how our students think?” Am. J. Phys. 82, 537551 (2014); doi: 10.1119/1.4874260.

9 C.E. Wieman, G.W. Rieger, and C.E. Heiner, "Physics Exams that Promote Collaborative Learning," The Physics Teacher 52, 51-53, (2014); doi: 10.1119/1.4849159. 